\title{
Large Scale Prediction of Protein Interactions by a SVM-Based Method
}

\author{
Francesco Marangoni ${ }^{1}$, Matteo Barberis ${ }^{1}$, and Marco Botta ${ }^{2}$ \\ 1 Fondazione per le Biotecnologie, Torino, Italy \\ 2 Dipartimento di Informatica, Università di Torino, Torino, Italy \\ botta@di.unito.it
}

\begin{abstract}
A number of techniques have been developed in order to address issues such as genome, trascriptome and proteome analysis. However, a time and cost effective technique for interactome analysis is still lacking. Lots of methods for the predicion of protein - protein interacions have been developed: some of them are based on high quality alignment of sequences, others are based on the tridimensional features of proteins, but they all bear strong limitations that make impossible their large scale application. Recently, an SVM-based machine learning approach has been used to address this topic. Although the method was able to correctly classify $80 \%$ of the test samples, it was not applied to the prediction of yet unknown interactions. In this work, we address this topic and show that an optimized, SVM-based machine learning approach trained with combinations of shuffled sequences as examples of lack of interaction is unable to make large scale predictions of interaction.
\end{abstract}

\section{Introduction}

The recent sequencing of human and other species genomes has given a strong impulse to the large scale study of genes and their products. A number of techniques have been developed in order to address issues such as genome, trascriptome and proteome analysis.

Unfortunately, and in contrast with other cell molecules, a bench technique that allows a large scale, cost and time effective analysis of interactome is still lacking. To overcome this limitation, several bioinformatic approaches have been developed to make predictions, which subsequently have to be validated by classical experiments of wet biology.

The search for pairs of proteins that interact is really a challenging issue. Given that a protein has from 2 to 10 interacting partners within a cell [5], it is clear that a bunch of interesting proteins has to be distinguished from a proteome of noisy ones.

Some bioinformatic approaches rely on the alignment of sequences coming from different organisms. The principal methods are philogenetic profiling, gene order conservation, "Rosetta stone", co-evolution analysis, in silico two hybrid [5] [8]. Even if these methods are considered preferable [1], they bear strong limitations. First of all, they need high quality sequences that are not always available. Moreover, they are based on the comparison of homolog sequences from different organisms. Inferring that an organism has (or has not) a homolog for a given sequence means that the organism has 
to be fully sequenced at a good qualitative standard. As a consequence, even if those methods provide good results, they can be hardly ever applied.

Other methods, namely the "docking" methods, try to infer an interaction by analysing the tridimensional complementarity of two proteins. The strongest limitation of such methods is that the tridimensional structure is known for a little minority of the proteins. This problem could be partially overcome by the so-called "threading" procedures, which combine homology modelling, structure refining and tridimensional complementarity analysis. Despite of this, the method cannot be applied to the sequences that lack structurally resolved homologs.

Recently, an SVM-based prediction method has been proposed that, looking exclusively at the primary structure and related physico-chemical properties would be functional and could be applied without limitations. Such method learns from examples of interacting and non - interacting proteins, represented as physico-chemical characteristic patterns, and tries to rule out the regularity (which is often unknown) that permits to discriminate between the two classes [2].

SVM is a supervised, kernel machine learning method that is based upon the concept of structural risk minimization [1]. It has recently been used for bioinformatic purposes [2] [9] because of some interesting features. First of all, it can efficiently manage huge amounts of data; then, the phenomenon of overfitting is strongly reduced; moreover it exists a publicly available implementation in $\mathrm{C}$ language which is computationally very efficient [4]. An SVM-based method has been used by Bock and Gough [2] to classify pairs of interacting and non-interacting proteins, with a precision of $80 \%$. It must be underlined here that, while interacting pairs of proteins are easily found in specialized databases, it does not exist a database of non-interacting proteins; then, negative examples were generated by shuffling the sequence of interacting proteins. The strongest limitation of this approach is that the authors did not apply the method to the real problem of predicting new interactions.

The present work has three purposes: to determine the crucial parameters for the construction of good SVM input vectors, to ameliorate the classification performance of $80 \%$ found by Bock and Gough, and to make predictions of novel interacting pairs of proteins through our optimized SVM-based method. The paper is organized as follows: in the next section a review of the methods used is given, followed by a summary of the obtained results, and finally a general discussion about the results is provided.

\section{Matherials and Methods}

Hardware and software. The computer used all over this work is an AMD DURON 750 $\mathrm{MHz}$, with $576 \mathrm{Mb}$ RAM. The software implementing the SVM algorithm was written in $\mathrm{C}$ language and kindly made accessible to the scientific community by Dr. T. Joachims [4]. All the other scripts needed for this work were developed in PERL 5.0 by the authors.

Databases and flatfiles. The Database of Interacting proteins (DIP) was used as source of information about interactions (24th January 2002 and 28th June 2002 releases) and sequences of interacting proteins (2nd June 2002 release). AAindex database provided the information about the aminoacid related physico-chemical properties ( $31 \mathrm{st}$ 
July 2002 release). Saccharomyces Genome Database (SGD) was the source of the Saccharomyces cerevisiae whole proteome file.

Sets of physico-chemical properties. Two indexes for each of the five major index classes were chosen on the basis of their completeness and the lack of correlation with the other chosen indexes. We chose DAYM780101 and HUTJ700101 for composition, SWER830101 and JANJ780101 for hydrophobicity, CHAM820101 and DAYM780201 for miscellaneous physico-chemical properties, GEIM800105 and NAGK730102 for beta-propension, GEIM800101 and NAGK730101 for alpha-and turn propension. Two sets of aminoacidic features were built choosing one index per class.

Generation of vectors representing interactions. The vectors representing the supposed interaction between two proteins were generated as described in [2]. Briefly, an aminoacid index was used to substitute the aminoacid residues of the primary sequence. This vector was standardized to a fixed length, and vectors originated from different indexes joined. Pairs of interacting proteins were represented by the joining of the two respective vectors. Negative examples were generated in the same way starting from shuffled sequences of interacting proteins.

Learning sets and Test sets. All learning sets were composed by a variable number of examples of interaction and the same number of examples of non-interaction. Where not otherwise specified, the test set was composed by the 2300 interactions that are uniquely found in the more recent release of DIP database, and 2300 examples of lack of interaction.

Output data analysis. Pairs of proteins were considered as interacting when SVM returned a value greater than zero; otherwise they were considered as non-interacting.

\section{Summary of Results}

In order to optimize the SVM-based classifier, we trained SVM with 2000 examples of interaction and 2000 examples of lack of interaction varying the standardization length of the vectors and the sets of physico-chemical properties. Length of 100, 200 and 400, and three sets of physico-chemical properties (Set 1 and Set 2 contained 5 indexes, and Set 3 contained all the ten indexes) were chosen. As shown in Fig. 1, we found that the classification performance grows when the standardization length grows as well.

Moreover, one of the 5-indexes set (Set 1) got better performances than the others in classifying examples of either interaction or non interaction. Surprisingly, Set 3, comprising all 10 indexes, failed to improve the classification performance: in fact, it gave an overall performance strictly similar to that obtained by the worst 5 -indexes set (Set 2).

Then, we studied the influence of the number of training instances on the classification performance. As shown in Fig. 2, the curves that link the number of learning examples to the classification performance were logarithmic-like for both instances of interaction and lack of interaction. Some events characterized the curves when $>500$ learning examples of interaction were used: both curves showed a very similar performance, the standard deviation of each point became extremely low, and a plateau was reached. We chose as optimal the number of 2200 learning examples of interaction, be- 


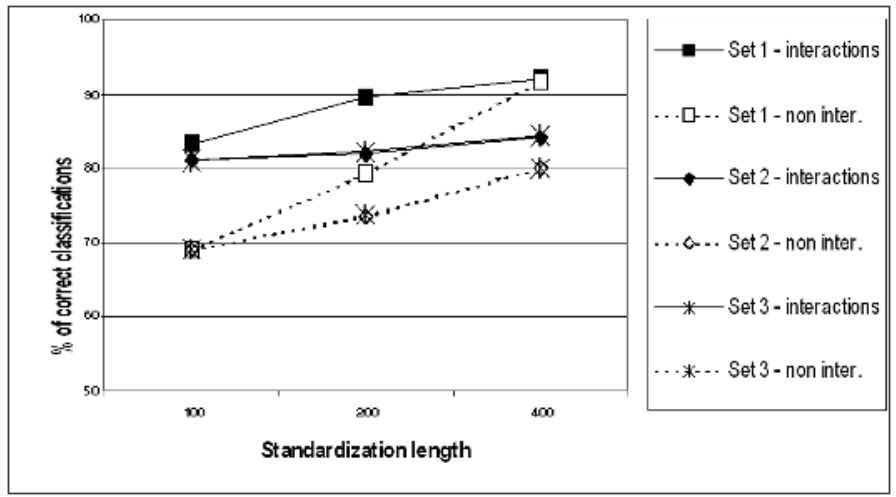

Fig. 1. Effect of the standardization length and of the three sets of physico-chemical aminoacidic features on the SVM-method classification performance. For every set, the classification performance was studied for both interacting and non-interacting pairs of proteins

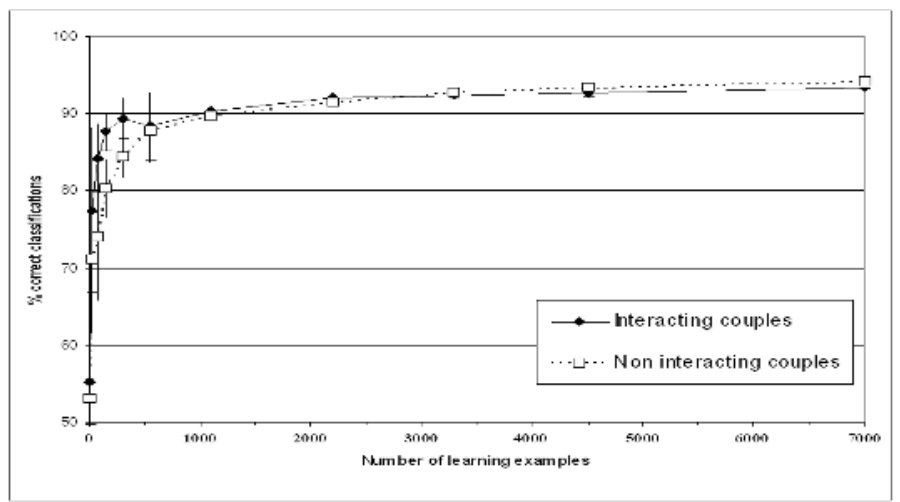

Fig. 2. Link between the number of learning examples used and the classification performance obtained. Every dot represents the average \pm standard deviation of 5 independent experiments

cause it corresponded to the plateau point that was computed using the smallest number of learning examples, thus using the lowest amount of computational resources.

The optimizing parameters were then applied to build a Saccharomyces cerevisiae restricted classifier. This classifier had a classification performance of $92 \%$ for both interacting and non-interacting proteins.

\section{Discussion}

Three parameters were individuated as crucial for the construction of interactionrepresenting vectors of good quality: the standardization length of the vectors, the set of physico-chemical properties, and the number of learning examples. 
Another crucial point was the right choice of examples of lack of interaction. Since a database of non-interacting protein does not exist, we needed to generate artificial examples of non-interacting proteins. Bock and Gough proposed that shuffled sequences from former interacting proteins could act as a non-interacting pair of proteins [2]. Even if it appears a very artificious method, we kept it for the present work.

As far as the standardization length is concerned, we found that the best performances were achieved with standardization length of 400, while lower lengths led to lower classification performances. The analysis of the classification errors occurring using a smaller standardization length clarified that errors were made mostly when classifying pairs of protein composed by at least one much bigger than the average (data not shown). This could lead to the hypothesis that "long" proteins constrained in "small" space lose the features needed for a correct classification.

Two sets of 5 physico-chemical properties were tested, and one of them gave performances reaching $92 \%$ correct classification rate. We then tried to join those sets, but unexpectedly we found that it had a classification performance tightly resembling that of the worst 5-properties set. An interference effect due to the redundancy of information was supposed, accordingly to Zavaljevski et al. [9], that reported an interference phenomenon on another application of SVM.

We noticed that the law that connects the number of learning examples to the classification performance is logarithmic-like and so it reaches a plateau. The best number of learning examples was determined to be the lowest computational effort needed to reach the best performance. We determined that the best amount of examples was 2200 for both interacting and non-interacting pairs.

We then moved to another topic, and tested whether our SVM-based classifier could be used as a predictor of protein interactions in a yeast model. We considered three Saccharomyces cerevisiae nuclear proteins involved in the yeast cell cycle: Sic1, Cdc28 and Clb5 [3] [6] [7], and we looked for their partners in the whole Saccharomyces cerevisiae proteome.

First of all, we needed to adapt the SVM-based classifier to an SVM-based predictor. This issue can be addressed by the Bayes' formula, in which the number of expected interactors and the total number of proteins are crucial. In our case, we had nearly 10000 proteins to test, among them we expected 10 interactors for each of our model proteins, according to Marcotte et al. [5]. It is immediately clear that the method in our hands would have given only $1.125 \%$ of overall correct predictions, while $8 \%$ of the whole proteome would have been proposed as interacting. Moreover, to obtain a prediction percentage $>50 \%$, we will need to build a classifier with a performance of $99.9 \%$ and a false discovery rate of $0.1 \%$.

Despite this, we applied our SVM-based predictor to the whole Saccharomyces cerevisiae proteome, in order to check if we could obtain the theoretical percentage of $8 \%$ of the whole proteome as being potentially interacting for each of our model proteins. Surprisingly, the SVM-based classifier recognized nearly all the proteome as being potentially interacting with the studied proteins. In particular, Sic1 was associated with $98.6 \%$, Clb5 with $96.0 \%$ and Cdc28 with $99.4 \%$ of the Saccharomyces cerevisiae proteome. 
One of the main reasons for this could be that our SVM-based predictor recognized real proteins from shuffled sequences, and did not distinguish interacting from noninteracting pairs of proteins. An evidence of this is that our predictor classified as noninteracting almost all the shuffled sequences we submitted (data not shown). Thus, in contrast to what Bock and Gough proposed, the assumption that shuffled sequences can well surrogate examples of lack of interaction appears here to be wrong.

Future research will be focused on further optimization of vector construction and on the generation of more reliable examples of lack of interaction. This could lead to the minimization of the false discovery rate, and finally to a reliable SVM-based method for the large scale prediction of protein interactions.

\section{Acknowledgements}

The authors wish to thank Dr. Elena Spoldi, Dr. Claudia Mondino and Dr. Ferdinando Di Cunto for the outstanding organization and scientific structure of the Master course in Bioinformatics, and Dr. Daniela Morale for the insightful discussions and help.

\section{References}

1. P. Baldi and S. Brunak, Bioinformatics: A machine learning approach. 2nd edition, MIT-Press (2002).

2. J.R. Bock and D.A. Gough, Predicting protein-protein interactions from primary structure Bioinformatics, 17 (2001) 455-460.

3. L. Dirick, T. Bšhm and K. Nasmyth, Roles and regulation of Cln-Cdc28 kinases at the start of the cell cycle of Saccharomyces cerevisiae, EMBO J., 14 (1995) 4803-4813.

4. T. Joachims, B. Sholkopf, C. Burges, and A. Smola, Making large-scale SVM learning practical, Advances in kernel methods - support vector learning MIT-Press (1999).

5. E.M. Marcotte, M. Pellegrini, H.L. Ng, D.W. Rice, T.O. Yeates, and D. Eisenberg, Detecting protein function and protein-protein interactions from genome sequences, Science, 285 (1999) 751-753.

6. M.D. Mendenhall, W. Al-jumaily, and T.T. Nugroho, The Cdc28 inhibitor p40Sic1 Prog. Cell Cycle Res., 1 (1995) 173-185.

7. E. Schwob and K. Nasmyth, CLB5 and CLB6, a new pair of of B cyclins involved in DNA replication in Saccharomyces cerevisiae Genes Dev., 7 (1994) 1160-1175.

8. A. Valencia and F. Pazos, Computational methods for the prediction of protein interactions, Curr. Opin. Struct. Biol., 12 (2001) 368-373.

9. N. Zavaljevski, F.J. Stevens, and J. Reifman, Support vector machines with selective kernel scaling for protein classification and identification of key amino acid positions, Bioinformatics, 8 (2002) 689-696. 